\title{
Miniaturized Coupling Structures for Decoupling PIFAs on Handheld Devices
}

\author{
Qian Li and Yaxin Yu \\ Electronics and Photonics Department, Institute of High Performance Computing, \\ $A^{*}$ STAR, Singapore 138632 \\ Correspondence should be addressed to Qian Li; benbenandlq@gmail.com
}

Received 21 September 2015; Revised 9 January 2016; Accepted 19 April 2016

Academic Editor: Yuan Yao

Copyright (C) 2016 Q. Li and Y. Yu. This is an open access article distributed under the Creative Commons Attribution License, which permits unrestricted use, distribution, and reproduction in any medium, provided the original work is properly cited.

\begin{abstract}
One efficient approach is introduced in this paper to reduce mutual coupling and correlation coefficient for two closely placed PIFAs in a handheld device. The approach is based on one miniaturized structure which consists of two metallic layers, printed on either side of one thin dielectric layer. Due to the small spacing between two conducting patches, high electromagnetic field is induced within the dielectric layer. The geometry and position of this structure have been modified to decouple the PIFA array at $1.9 \mathrm{GHz}$ and produce maximum miniaturization thereby occupying less space on a handheld device ground plane. By employing the proposed structures, a $20 \mathrm{~dB}$ reduction in mutual coupling is achieved. The correlation coefficient also reduces to 0.007278 . The performance of the structure is validated by both simulated results and measured data obtained from several fabricated prototypes.
\end{abstract}

\section{Introduction}

Nowadays, the huge demands for data rate result in serious technology challenges for a next generation wireless communication system. As one solution, Multiple-Input-MultipleOut (MIMO) has been proposed as an efficient method to increase wireless channel capacity by using multiple antenna elements without additional spectrum and power [1]. Nonetheless, in a compact mobile terminal, the limited interval will produce high mutual coupling and correlation coefficient between adjacent antenna ports and eventually degrade the system performance [2]. As such, in recent years, some researches have been presented to reduce mutual coupling effects and correlation coefficient, especially for compact mobile terminal. In [3], a spiral defected microstrip structure is employed to significantly reduce the mutual coupling for patch antenna array. A waveguide metamaterial realized by crossed-meander-line slits is proposed as an efficient method to mitigate the mutual coupling problem in [4]. Another technique, based on folded split-ring resonators (FSRRs), is proposed to enhance isolation between antenna elements in [5]. Recently, a novel miniaturized Complementary Metallodielectric Electromagnetic Band Gap (CMEBG) structure was presented to suppress surface wave propagation and to offer a small structural footprint thereby occupying very little space on a compact ground plane [6]. Also, the complex DGS (Defected Ground Structure) has been investigated for different antenna types [7, 8].

Based on these researches, in order to further miniaturize the dimension of the structure, one novel miniaturized double-layered structure is proposed for decoupling PIFA MIMO array in a handheld device in this paper. Here, one conducting patch is employed for placement on ground plane; strong coupling is produced within the separation region thus resulting in the double-layered structure. Therefore, one microstrip line excitation is initially employed, compared to any complex antenna array, for analyzing the stop-band/band gap properties of the proposed structures. By appropriately optimizing the dimensions and position of the structure, high mutual coupling between two PIFA elements has been restrained. Moreover, the proposed structures occupy small space, allowing for the constraints of limited space and increasing flexibility in their applications for other electronic components installment. Fabricated prototypes have been experimentally tested and the measurement results are presented, validating the simulated designs. 


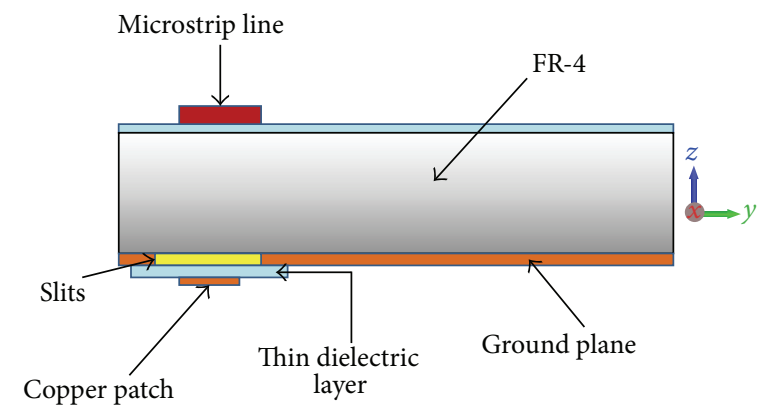

FIgURE 1: Schematic view of double-layered structure with microstrip line.

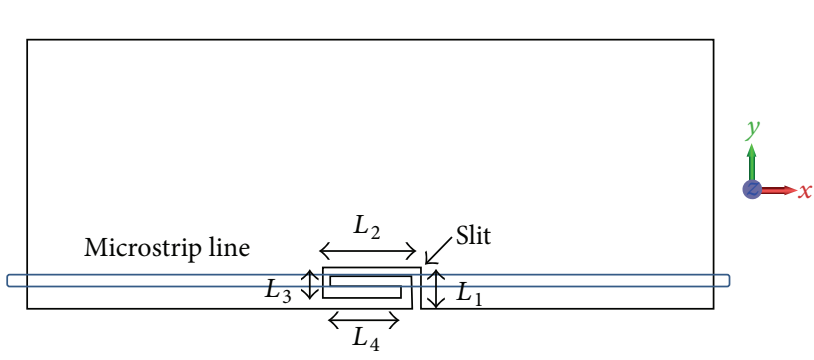

(a)

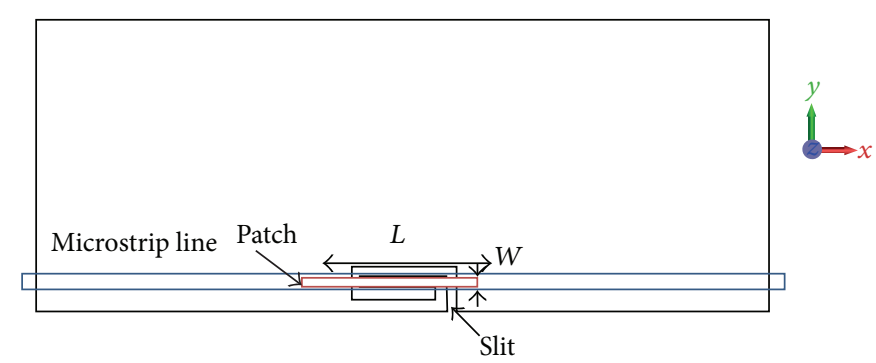

(b)

FIGURE 2: (a) Single convoluted slit is inserted in the ground plane. (b) Proposed convoluted double-layered structure.

\section{Double-Layered Structure Design}

The configuration of designed double-layered structure with one microstrip line is presented in Figure 1. Two metallic layers are printed on either side of one thin dielectric structure. Due to small thickness of dielectric structure (55 $\mu \mathrm{m})$, high EM coupling is induced between the two metallic layers $[9,10]$. The function of the proposed structures could be described using an equivalent circuit of capacitive and inductive loads between the ports. A parallel LC circuit approximately describes one unit cell (single resonator) of the double-layered structure. The resonant frequency of the single double-layered resonator can be obtained from [11]:

$$
f=\frac{\omega}{2 \pi}=\frac{1}{2 \pi \sqrt{L C}}
$$

where $L$ is the inductance produced by the currents flowing on the patches and ground plane. The capacitance $C$ of the structure is predominantly produced by the two closely spaced parallel conducting elements separated by distance $d$ and is obtained from

$$
C=\varepsilon_{r} \varepsilon_{0} \frac{A}{d}
$$

where $A$ is the overlapping area of the conducting elements, $\varepsilon_{r}$ is the relative static permittivity, and $\varepsilon_{0}$ is the free space permittivity. The distance $d$ between the two layers remains the same $(55 \mu \mathrm{m})$ for all designs in this paper and is small enough to produce high capacitance. Also, when the width of the conductor patches increases, the overlap area $A$ will increase and result in a high value of capacitance. Therefore,
TABLE 1: Dimensions of the proposed structure.

\begin{tabular}{lccccccc}
\hline Parameters & $L_{1}$ & $L_{2}$ & $L_{3}$ & $L_{4}$ & Sw & Pw & Pl \\
\hline Values/mm & 6 & 14 & 5 & 10 & 1.61 & 15.5 & 0.5 \\
\hline
\end{tabular}

the resonance is expected to move towards lower frequencies. It is expected that the band gap could be shifted by changing the width of the conducting patches. The bandwidth can be calculated from the formula given below and corresponds to the width of the surface wave band gap [11]:

$$
\mathrm{BW}=\frac{1}{\mathrm{Q}}=\frac{\sqrt{L / C}}{\sqrt{\mu_{0} / \varepsilon_{0}}} .
$$

When the area of conducting patch increases, the higher capacitance causes the stop-band bandwidth to reduce.

In order to verify the stop-band effect of the doublelayered structure, one microstrip line is printed on a $100 \mathrm{~mm}$ $\times 40 \mathrm{~mm} \times 1.5 \mathrm{~mm}$ polyester dielectric sheet and placed at the top of a FR-4 dielectric substrate for excitation. Initially, one slit is meandered thereby increasing the electrical length and takes up less area on the ground plane shown in Figure 2(a). The convoluted shaped slit is inserted into the ground plane and optimized to resonance at $1.7 \mathrm{GHz}$. Also, one $15.5 \mathrm{~mm}$ length and $0.5 \mathrm{~mm}$ width conducting patch is placed on the slit, thereby forming the proposed doublelayered coupling structure, as depicted in Figure 2(b). The geometry with detailed dimensions of the double-layered structure is illustrated in Table 1.

The effect of the proposed double-layered structure is analyzed through simulated $S_{21}$ response with different patch 


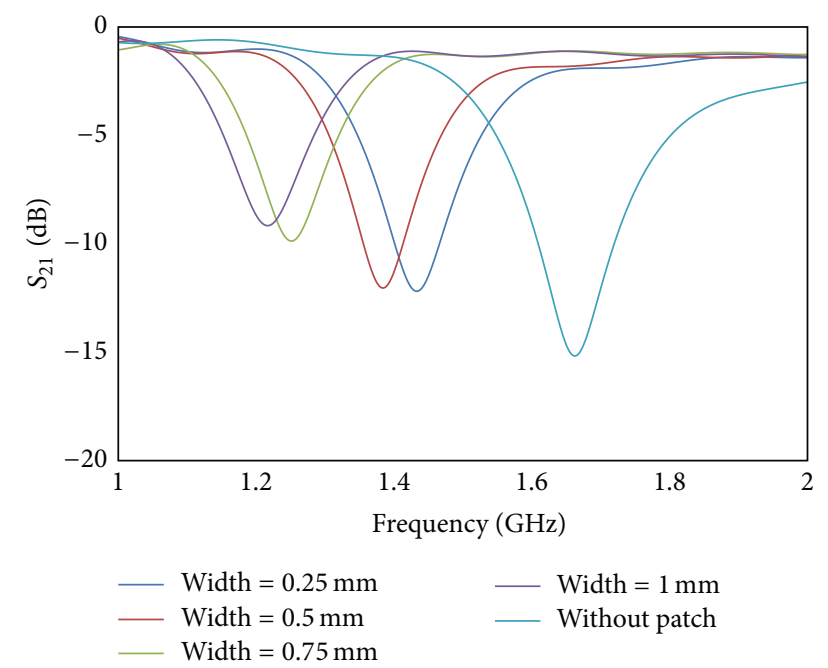

FIGURE 3: Simulated $S_{21}$ with different width of conducting patch.

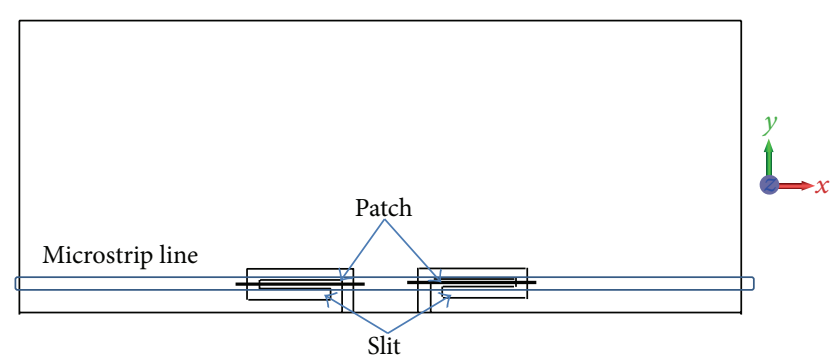

FIGURE 4: Two identical double-layered structures implemented on the ground plane.

width. As depicted in Figure 3, it is obvious that the proposed double-layered structure acts as a band-stop filter. With the width varying from $0.25 \mathrm{~mm}$ to $1 \mathrm{~mm}$, the resonant frequency of $S_{21}$ shifts from $1.43 \mathrm{GHz}$ to $1.2 \mathrm{GHz}$. This design occupies a small footprint near the edge of the ground plane which can be advantageous for use in compact devices with a large number of electronic components installed on the ground plane. However, after employing the double-layered coupling structure, the increase in the capacitance results in the bandwidth decreasing when the overlapping area is increasing, as expected above.

In order to obtain more bandwidth, two identical structure elements are employed, as shown in Figure 4. The dimensions of the structure elements are kept the same as before. The spacing between two structures is $8 \mathrm{~mm}$. As represented in Figure 5, the cut-off frequency of the band gap slightly decreased. However, it is evident that the bandwidth increases considerably after employing two structure elements. These simulated results are encouraging since the proposed doublelayered structure is able to support an expected bandwidth by increasing the number of the proposed structure elements.

\section{Decoupling PIFA Array}

A model of PIFA array, designed for handheld devices, is illustrated in Figure 6. The antennas are mounted on a

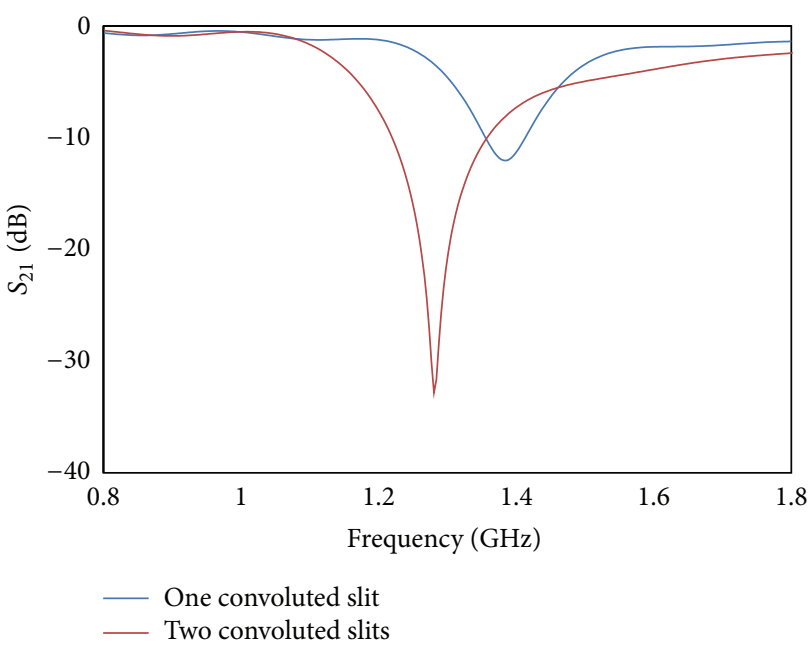

FIGURE 5: Simulated $S_{21}$ with one or two double-layered structures.

TABLE 2: Dimensions of the proposed structure.

\begin{tabular}{lccccccc}
\hline Parameters & $L_{1}$ & $L_{2}$ & $L_{3}$ & $L_{4}$ & Sw & Pw & Pl \\
\hline Values/mm & 4 & 10.7 & 3 & 8.5 & 1.61 & 15.5 & 0.5 \\
\hline
\end{tabular}

$100 \mathrm{~mm} \times 40 \mathrm{~mm}$ FR-4 dielectric substrate, which has a thickness of $1.2 \mathrm{~mm}$ and a relative permittivity of 4.5 . The PIFA elements are designed to operate at $1.9 \mathrm{GHz}$. At this frequency, the two PIFAs are approximately half-wavelength apart $(90 \mathrm{~mm})$ and exhibit an $S_{21}$ of about $-10 \mathrm{~dB}$, as shown in Figure 7(a). In order to reduce the mutual coupling, the double-layered structure is implemented, as shown in Figure 6(a). The detailed size of the structure is presented in Table 2. By employing two double-layered structures on either side of the ground plane, a sharp transmission minimum is obtained at $1.88 \mathrm{GHz}$ with $S_{21}$ reduced to $-27 \mathrm{~dB}$ as shown in Figure 7(b). Both antennas remain well matched at the desired frequency where the double-layered structure resonates. As expected, the bandwidth of $S_{21}$ does not accommodate the resonant frequency of the antennas. To obtain the desired bandwidth, four coupling structure elements are employed and placed between two PIFAs. As presented in Figure 6(b), the separation between two slits is $10.25 \mathrm{~mm}$, and the distance between two patch edges is $5 \mathrm{~mm}$. Figure $7(\mathrm{c})$ illustrates the measured $S$-parameter of PIFA array with four coupling structures. At the resonance of the double-layered structures $(1.9 \mathrm{GHz})$, both antennas are matched $\left(S_{11}<\right.$ $-10 \mathrm{~dB}$ and $S_{22}<-10 \mathrm{~dB}$ ). Furthermore, the measured $S_{21}$ in the presence of four coupling structures decreases to $-35 \mathrm{~dB}$ at $1.9 \mathrm{GHz}$. The measured bandwidth $(-15 \mathrm{~dB})$ of $S_{21}$ increases to $150 \mathrm{MHz}$ which is much better than the original design.

In order to know the mechanism of the proposed structure, the surface current of the PIFA array is presented in Figure 8. When the antenna array is placed on a traditional ground plane, it can be observed that high concentration of current is induced on the antenna element on the right when the left antenna element is excited, as illustrated in Figure $8(a)$, thus producing high mutual coupling. By employing the double-layered structure, the surfaced current 


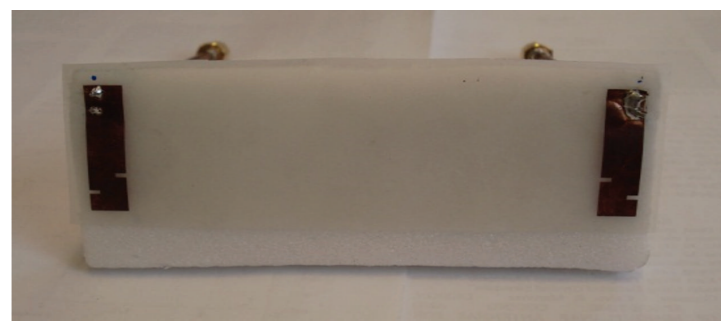

(a)
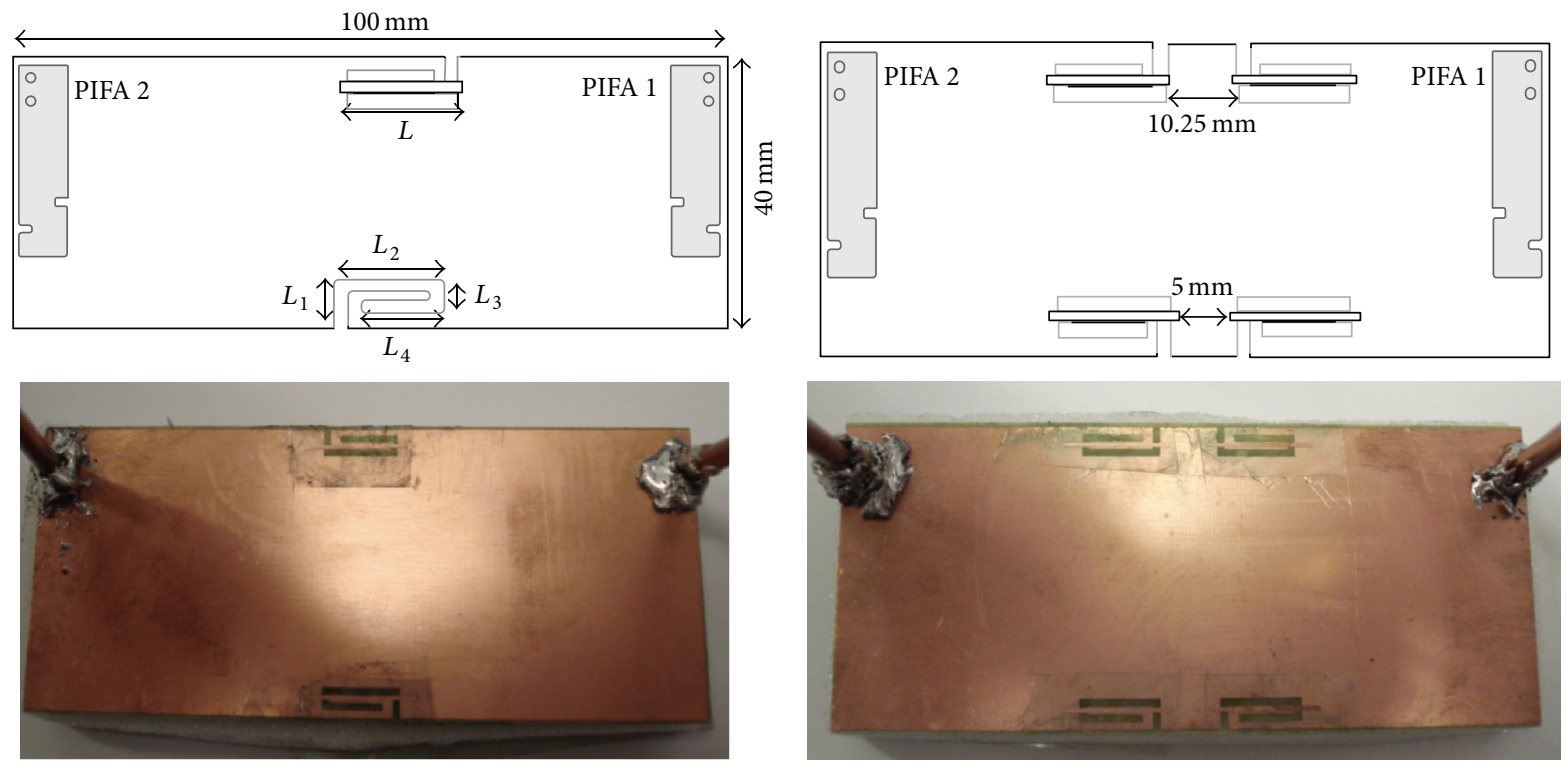

(b)

(c)

FIgURE 6: (a) Two-element PIFA array with (b) two convoluted double-layered structures and (c) four convoluted double-layered structures.

is trapped thus resulting in less induced current on the right antenna, as shown in Figure 8(b). Therefore, a mutual coupling reduction between two PIFAs has been obtained. This is attributed to the stop-band effect of the double-layered structures. The radiation patterns of the proposed UWB antenna array for the two cases are presented in Figure 9. As the surface current which is distributed on the ground is not symmetric along the $y-z$ plane, after the proposed structures are employed, the far field has been affected. However, as the coupling between antennas reduced, the gain has been improved.

In order to investigate the correlation between two antennas, based on the $S$-parameter, the correlation coefficient is calculated by a formula [12]:

$$
\rho_{\mathrm{e}}=\frac{\left|S_{11}^{*} S_{12}+S_{21}^{*} S_{22}\right|^{2}}{\left(1-\left(\left|S_{11}\right|^{2}+\left|S_{21}\right|^{2}\right)\right)\left(1-\left(\left|S_{12}\right|^{2}+\left|S_{22}\right|^{2}\right)\right)} .
$$

The correlation coefficient of all cases, namely, the antenna array without and with two and four double-layered structures, is presented in Figure 10. Compared with a convolutional ground plane, the correlation coefficient is significantly reduced by employing the double-layered structures. It is obvious that one minimum value of less than 0.007278 is obtained after the four double-layered structures are implemented. It is a very reasonable efficiency value and demonstrates that the mutual coupling reduction is not due to losses. Also, the total efficiency has been calculated and presented in Figure 11. With more proposed structures having been employed, the total efficiency increases at the resonated frequency.

\section{Conclusions}

A novel approach for mutual coupling reduction is presented by using double-layered coupling structures. A LC circuit description was analyzed for a qualitative explanation of the proposed structure. Simulated $S$-parameters as well as measurements of fabricated prototypes are presented. The two and four structure elements are employed to produce a $20 \mathrm{~dB}$ reduction in mutual coupling for the PIFAs array. The $S$-parameter, surface current, and correlation coefficient were studied, validating the effect of the proposed structures. Also, the proposed structures which have small footprint are positioned close to the edges of the ground plane thereby occupying less space.

\section{Competing Interests}

The authors declare that there are no competing interests regarding the publication of this paper. 

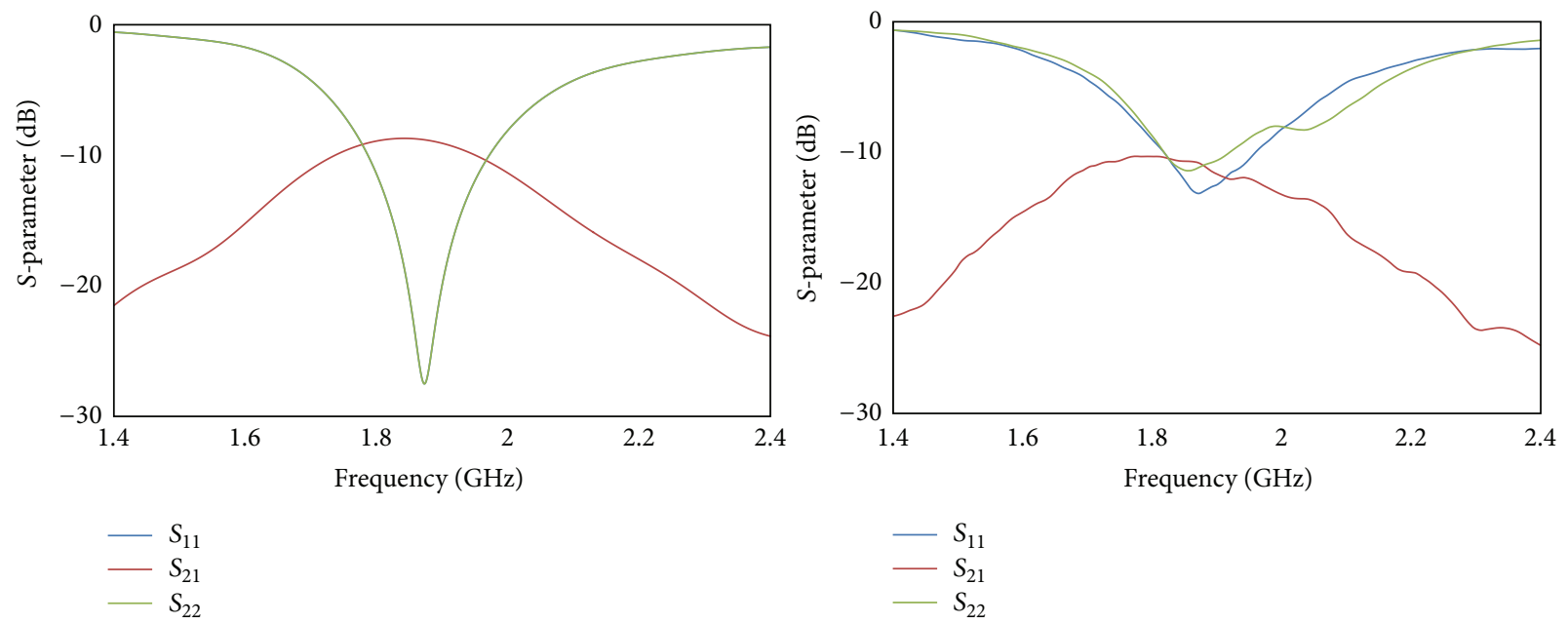

(a)
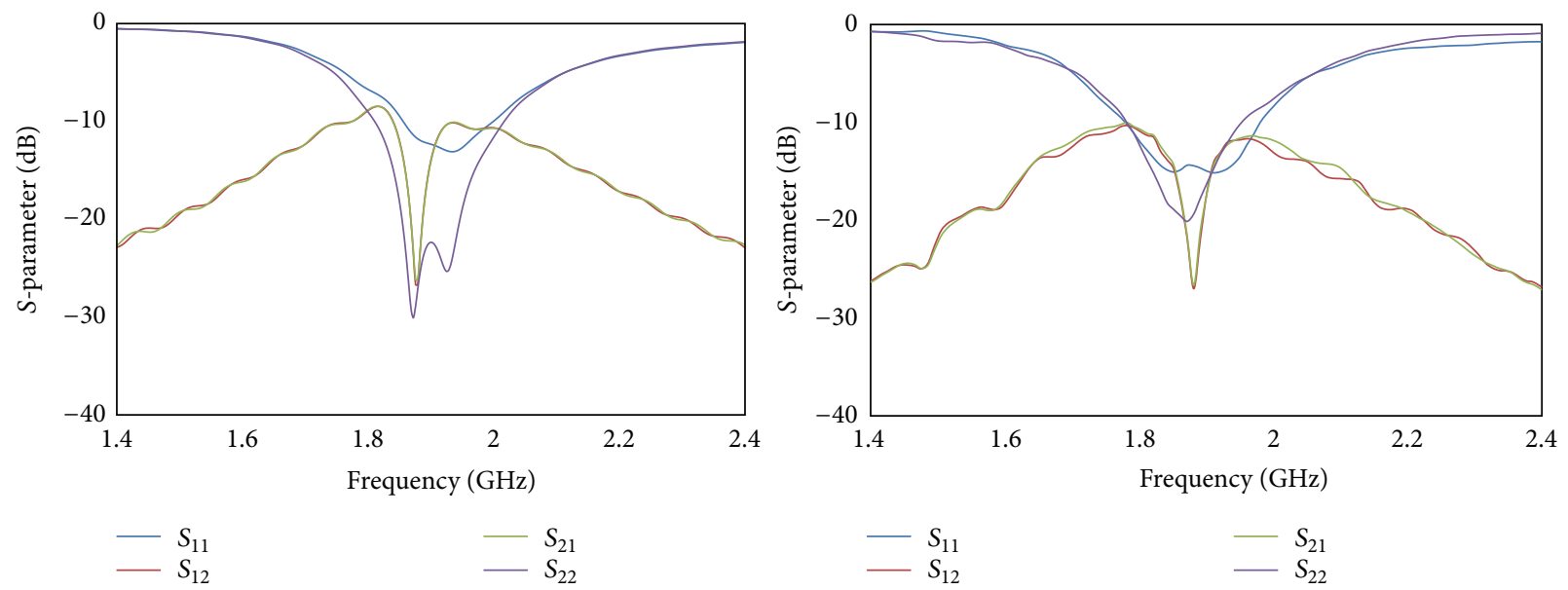

$-S_{11}$
$-S_{12}$

$-S_{21}$
$-S_{22}$

(b)
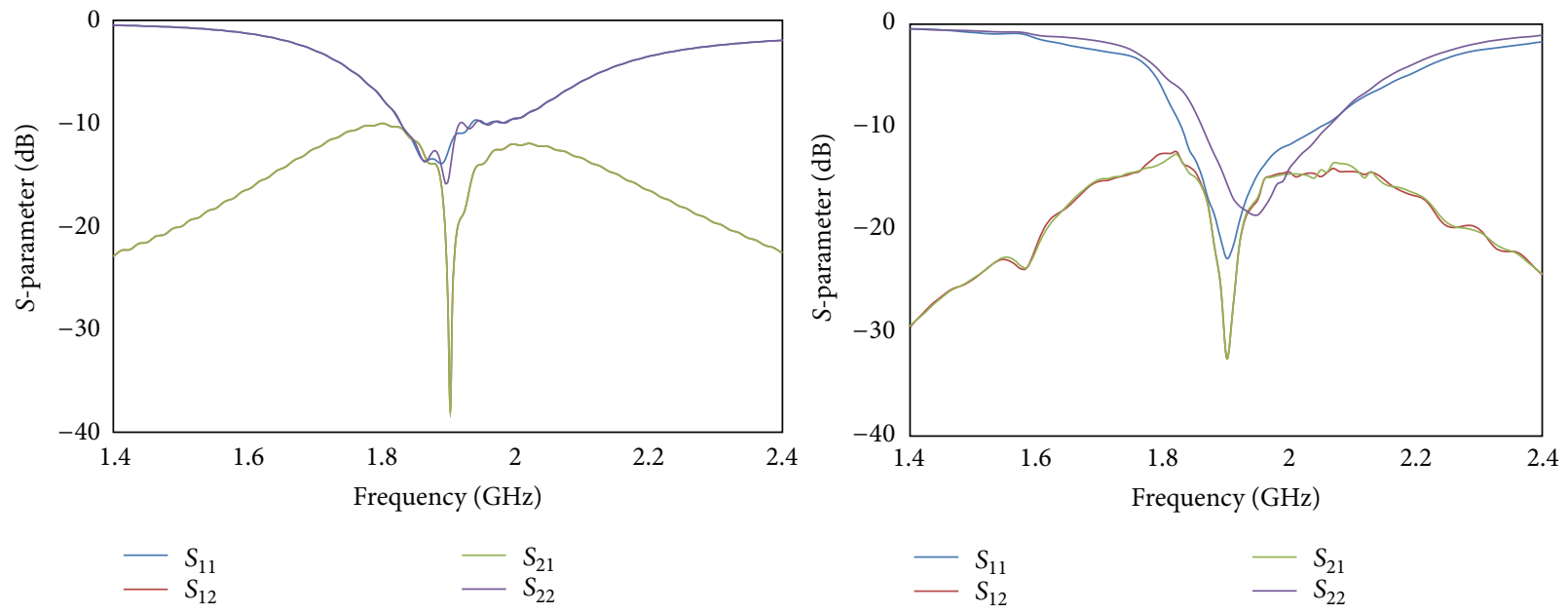

$-S_{21}$
$-S_{22}$

(c)

FIGURE 7: Simulated and measured S-parameters of the PIFA array (a) without and (b) with two and (c) with four double-layered structures. 

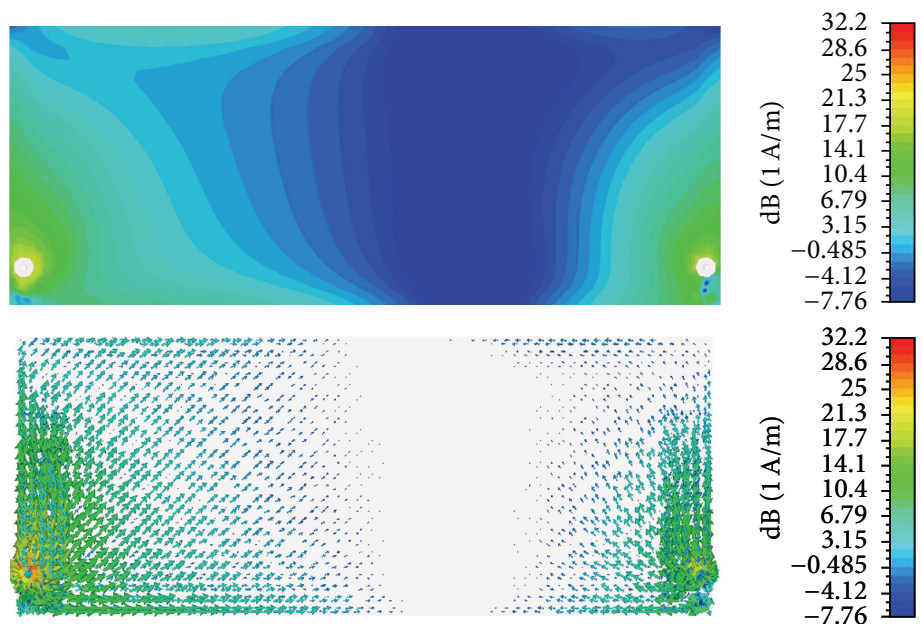

(a)
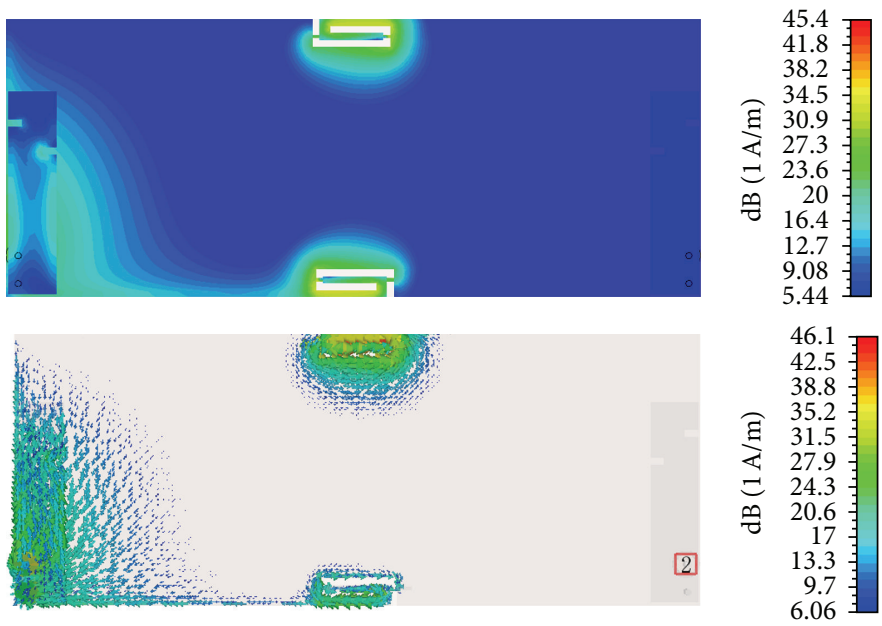

(b)

FIGURE 8: Current distributions of the two PIFAs (a) without and (b) with two double-layered structures.

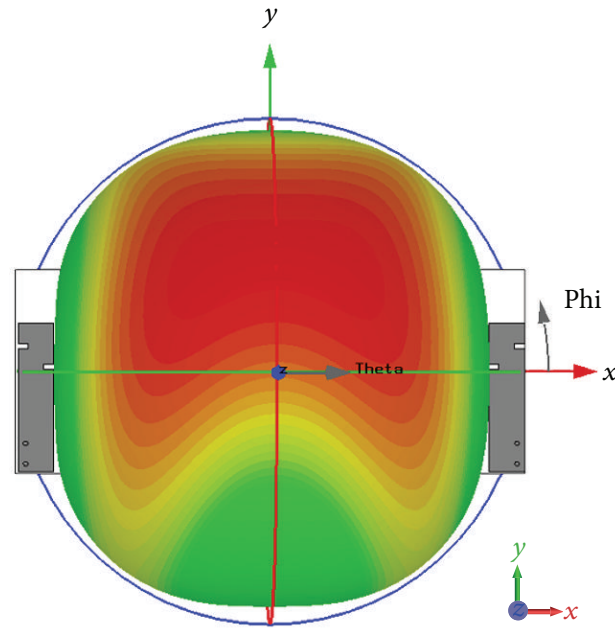

(a)

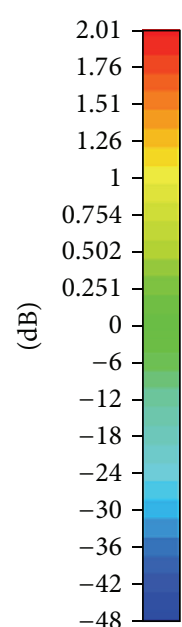

$-48$

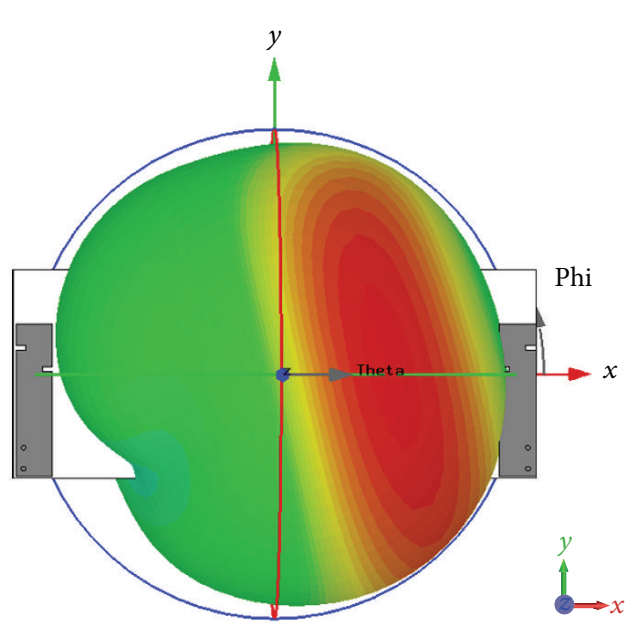

(b)

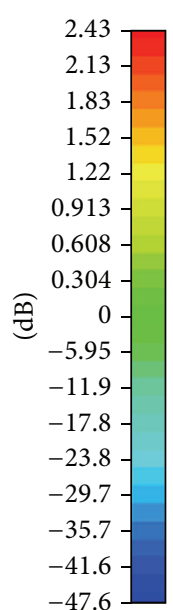

FIgURe 9: Far field of the two PIFAs (a) without and (b) with two double-layered structures. 


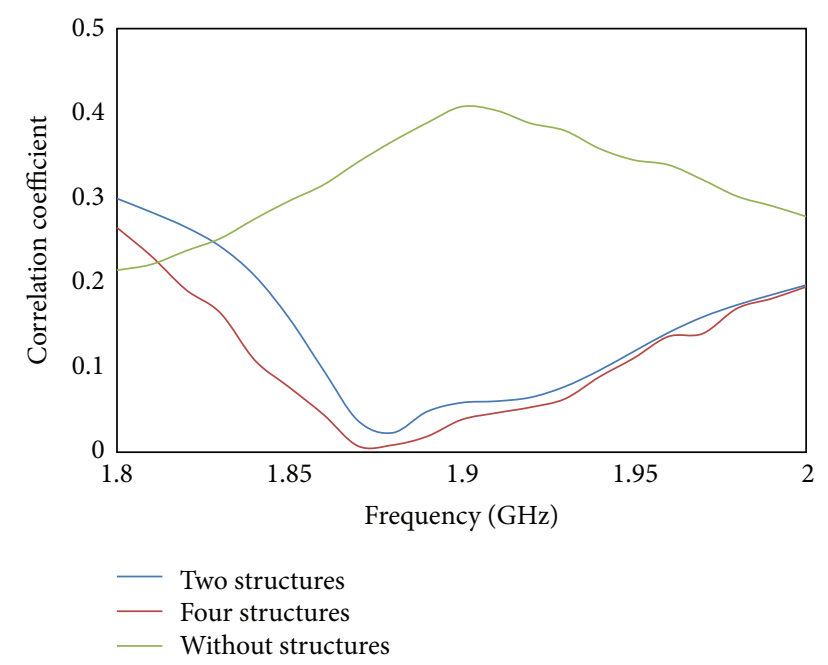

FIgURE 10: Correlation coefficient between two PIFAs without and with two slits or four slits.

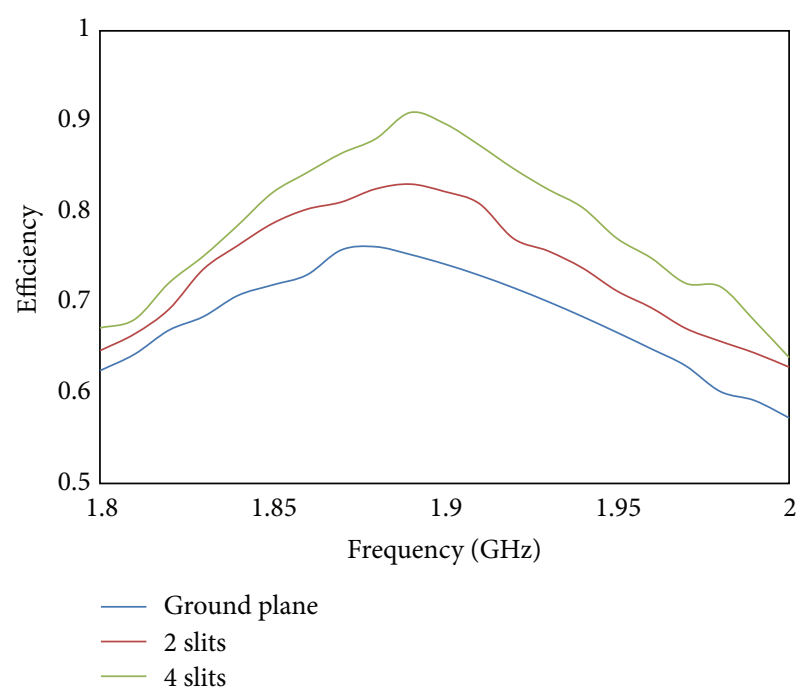

FIGURE 11: Total efficiency of PIFAs without and with two slits or four slits.

\section{References}

[1] J. W. Wallace, M. A. Jensen, A. L. Swindlehurst, and B. D. Jeffs, "Experimental characterization of the MIMO wireless channel: data acquisition and analysis," IEEE Transactions on Wireless Communications, vol. 2, no. 2, pp. 335-343, 2003.

[2] S. Lu, H. T. Hui, and M. Bialkowski, "Optimizing MIMO channel capacities under the influence of antenna mutual coupling," IEEE Antennas and Wireless Propagation Letters, vol. 7, pp. 287290, 2008.

[3] S. Fallahzadeh, H. Bahrami, A. Akbarzadeh, and M. Tayarani, "High-isolation dual-frequency operation patch antenna using spiral defected microstrip structure," IEEE Antennas and Wireless Propagation Letters, vol. 9, pp. 122-124, 2010.

[4] M. U. Khan and M. S. Sharawi, "Isolation improvement using an MTM inspired structure with a patch based MIMO antenna system," in Proceedings of the 8th European Conference on Antennas and Propagation (EuCAP '14), pp. 2718-2722, IEEE, The Hague, The Netherlands, April 2014.
[5] A. Habashi, J. Nourinia, and C. Ghobadi, "Mutual coupling reduction between very closely spaced patch antennas using low-profile folded split-ring resonators (FSRRs)," IEEE Antennas and Wireless Propagation Letters, vol. 10, pp. 862-865, 2011.

[6] G. Goussetis, A. P. Feresidis, G. Apostolopoulos, and J. C. Vardaxoglou, "Miniaturisation of defected ground plane using complementary metallodielectric electromagnetic band gap structures," in Proceedings of the IEEE International Workshop on Antenna Technology: Small Antennas and Novel Metamaterials (IWAT '06), New York, NY, USA, March 2006.

[7] M. Karakoikis, C. Soras, G. Tsachtsiris, and V. Makios, "Compact dual-printed inverted-F antenna diversity systems for portable wireless devices," IEEE Antennas and Wireless Propagation Letters, vol. 3, no. 1, pp. 9-14, 2004.

[8] C.-Y. Chiu, C.-H. Cheng, R. D. Murch, and C. R. Rowell, "Reduction of mutual coupling between closely-packed antenna elements," IEEE Transactions on Antennas and Propagation, vol. 55, no. 6, pp. 1732-1738, 2007.

[9] A. P. Feresidis, G. Apostolopoulos, N. Serfas, and J. C. Vardaxoglou, "Closely coupled metallodielectric electromagnetic band-gap structures formed by double-layer dipole and tripole arrays," IEEE Transactions on Antennas and Propagation, vol. 52, no. 5, pp. 1149-1158, 2004.

[10] G. Apostolopoulos, A. P. Feresidis, G. Goussetis, and J. C. Vardaxoglou, "Complementary metallo-dielectric electromagnetic band gap structures," in Proceedings of the European Microwave Conference, vol. 2, Paris, France, October 2005.

[11] D. S. Lockyer and J. C. Vardaxoglou, "Reconfigurable FSS response from two layers of slotted dipole arrays," Electronics Letters, vol. 32, no. 6, pp. 512-513, 1996.

[12] S. Blanch, J. Romeu, and I. Corbella, "Exact representation of antenna system diversity performance from input parameter description," Electronics Letters, vol. 39, no. 9, pp. 705-707, 2003. 


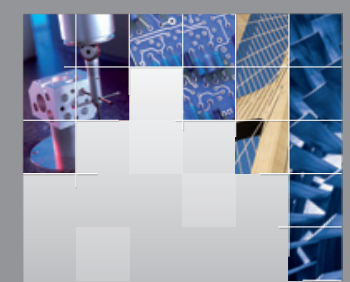

\section{Enfincering}
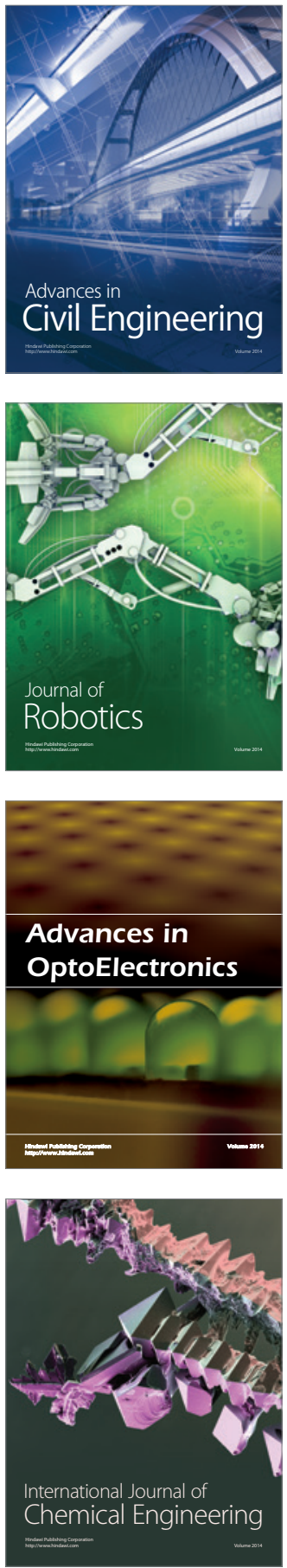

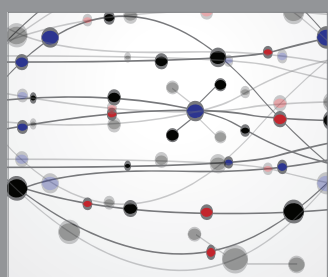

The Scientific World Journal

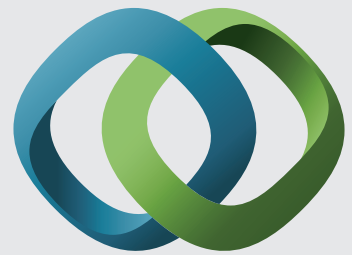

\section{Hindawi}

Submit your manuscripts at

http://www.hindawi.com
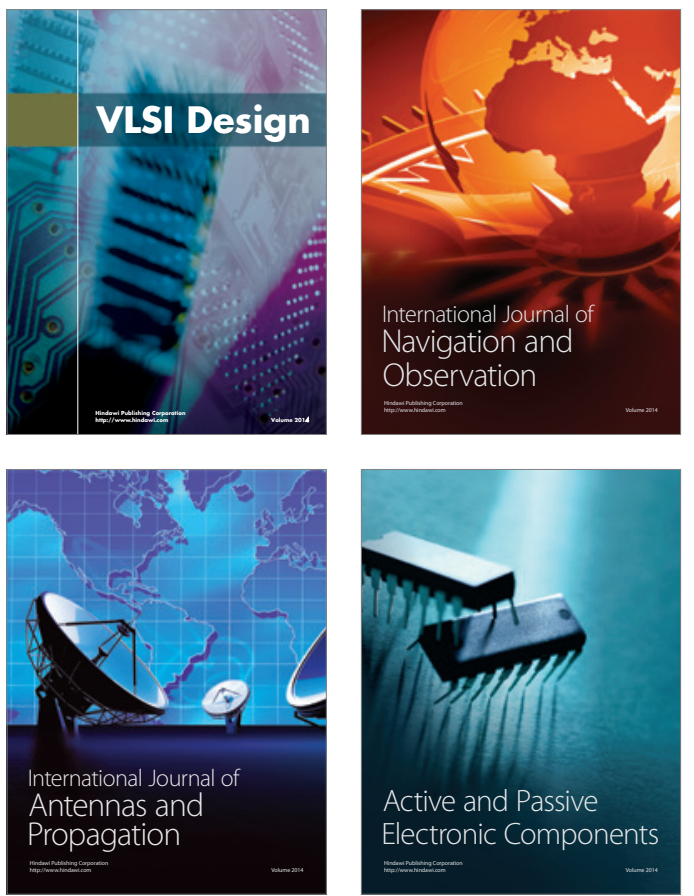
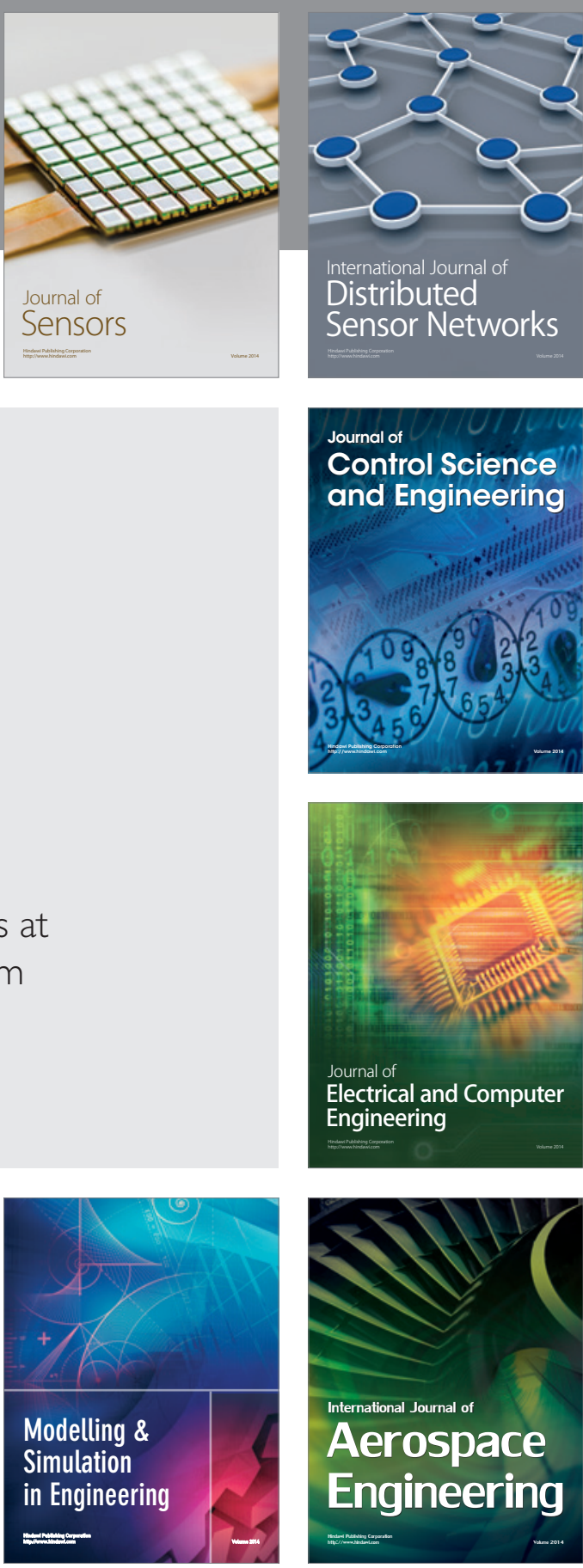

International Journal of

Distributed

Sensor Networks

Journal of

Control Science

and Engineering
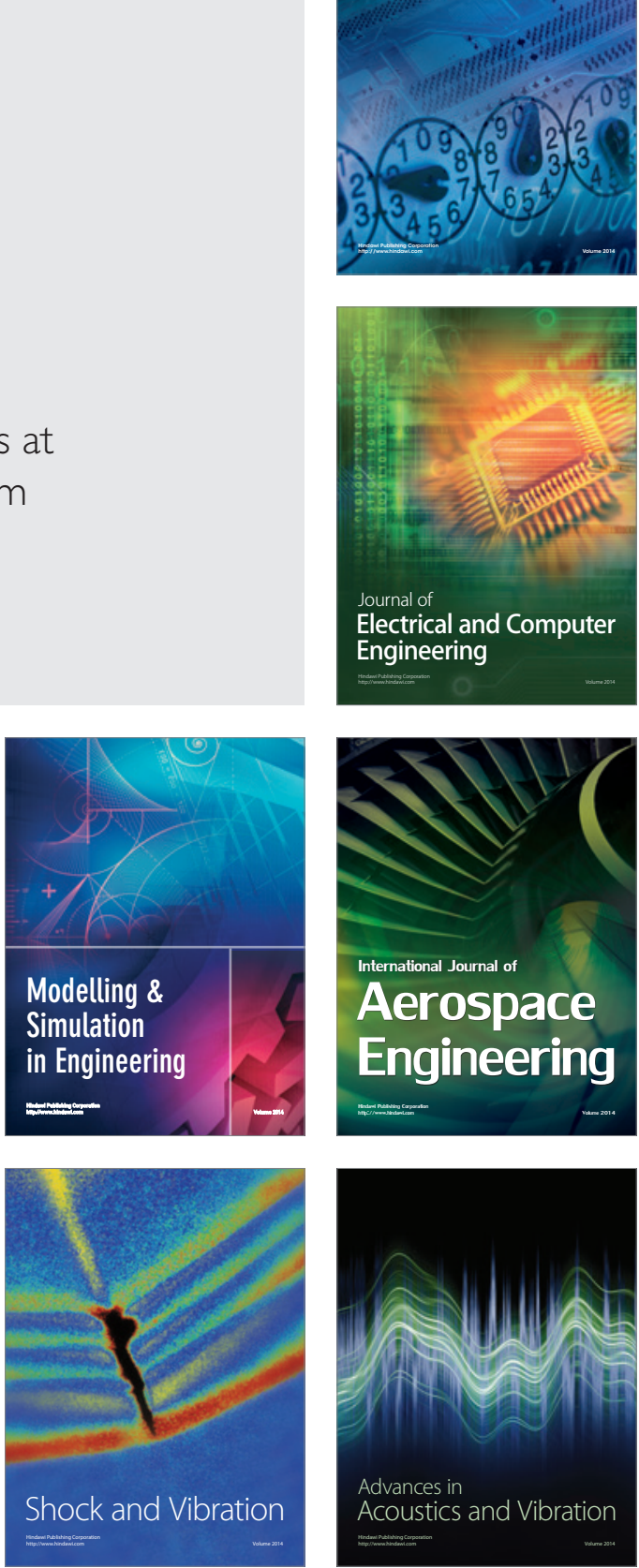